\title{
Les réfugiés du « Printemps des peuples » à
} Londres

Refugees of the 'Peoples' Spring' in London

Fabrice Bensimon

\section{OpenEdition}

\section{Journals}

Édition électronique

URL : http://journals.openedition.org/rfcb/1602

DOI : $10.4000 /$ rfcb.1602

ISSN : 2429-4373

Éditeur

CRECIB - Centre de recherche et d'études en civilisation britannique

\section{Édition imprimée}

Date de publication : 1 septembre 2003

ISSN : 0248-9015

\section{Référence électronique}

Fabrice Bensimon, «Les réfugiés du «Printemps des peuples » à Londres », Revue Française de Civilisation Britannique [En ligne], XII-3 | 2003, mis en ligne le 01 septembre 2003, consulté le 07 mai 2019. URL : http://journals.openedition.org/rfcb/1602 ; DOI : 10.4000/rfcb.1602

Ce document a été généré automatiquement le 7 mai 2019.

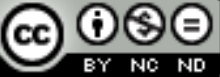

Revue française de civilisation britannique est mis à disposition selon les termes de la licence Creative Commons Attribution - Pas d'Utilisation Commerciale - Pas de Modification 4.0 International. 


\title{
Les réfugiés du « Printemps des peuples » à Londres
}

\author{
Refugees of the 'Peoples' Spring' in London
}

\author{
Fabrice Bensimon
}

1 La population dont il est question ici est « aux marges de la ville victorienne », mais d'une façon un peu particulière; c'est un groupe plus réduit que ceux qui sont étudiés dans d'autres articles. C'est, pourrait-on dire, une communauté marginale par accident: les réfugiés des révolutions de 1848-9, auxquels on peut ajouter les proscrits du 2 Décembre 1851. C'est un groupe dont les contours ont été peu cernés. On connaît Karl Marx, le plus célèbre de ces réfugiés, et quelques autres : le Hongrois Kossuth, l'Italien Mazzini, le Russe Herzen, les Français Louis Blanc et Ledru-Rollin, sans parler d'un autre réfugié illustre, qui n'était alors pas à Londres, Victor Hugo. On utilise les textes des uns et des autres. En revanche, il y a les milliers d'anonymes, de militants qui ont fui l'échec des révolutions de 1848-9, la période de réaction qui les a suivies, et ont vécu, parfois survécu, quelque temps à Londres, avant que de nouvelles conditions politiques leur permettent de retourner au pays, ou, pour certains d'entre eux, de gagner l'Amérique.

2 Il y a eu, somme toute, assez peu d'études sur ces réfugiés. Il y a plus de vingt ans, Bernard Porter consacra un ouvrage à leur place dans la vie politique anglaise des années $1850^{1}$. Et puis, récemment, plusieurs chercheurs se sont attachés à retracer leurs itinéraires, en particulier à la lumière des problématiques de l'histoire de l'exil'2.

Il est bien sûr impossible ici de dresser un tableau exhaustif de cette population. On voudrait surtout faire connaître les travaux récents sur ce groupe, en évoquant en particulier une communauté, celle des Français. D’abord, qui sont-ils? Puis, on évoquera les raisons pour lesquelles le pouvoir britannique les accueille, et l'attention qu'il leur consacre. On s'attachera ensuite à leur vie sociale et politique, en particulier à Londres. Enfin, on s'intéressera aux regards croisés entre eux et la société anglaise. 


\section{Les réfugiés à Londres}

4 Première interrogation, donc, qui sont ces réfugiés? Tout au long du XIXe siècle, la Grande-Bretagne accueille des réfugiés du continent. Des émigrés de la Révolution française aux Communards de 1871, de Charles X à Louis-Napoléon Bonaparte, en passant par Louis-Philippe, Guizot et Metternich - la liste est longue de ceux qui sont allés outreManche pour échapper à la répression des pouvoirs autoritaires, ou aux révolutions. Ils s'ajoutent aux nombreux immigrés économiques : les musiciens italiens, les marchands et les banquiers allemands, les professeurs de français, etc. - en tout quelque 50000 étrangers au milieu du siècle. Les réfugiés ne représentent qu'une petite minorité de cette population : en 1852, environ 4500 Français, 1300 Allemands, 800 Polonais, et quelques centaines de Russes, de Tchèques, d'Italiens, etc. - au total, jamais plus de 10000 , en 1850-1, et moitié moins après 1852. Ce groupe décroît au cours des années 1850, et dans les années 1860 , les exilés ne constituent plus guère un groupe à proprement parler, du moins sur le plan politique. L'essentiel de ces réfugiés sont à Londres, $80 \%$ des Français par exemple. Pour l'essentiel, ils se regroupent autour de Soho et de ses rues misérables d'alors. Les plus cossus vivent dans le West End, mais ils sont une minorité. Si Jersey et Guernesey sont des lieux connus de l'exil, les îles Anglo-Normandes n'attirent guère plus de quelques dizaines de réfugiés.

5 Pour la plupart, ces exilés vivent difficilement. De toute façon, la Grande-Bretagne n'est pas un vrai choix: c'est une destination imposée car, en Europe, pour des raisons sur lesquelles on reviendra, seule la Grande-Bretagne accueille alors les réfugiés de façon durable et systématique. Pour un exilé fortuné, comme Ledru-Rollin, il y a des milliers de nécessiteux, qui survivent difficilement, grâce à des petits boulots, à la mendicité, à la solidarité et à l'entraide, car d'anciens chartistes comme Ernest Jones et Julian Harney mettent en place des réseaux de secours. Ceux qui ont certaines qualifications (les cordonniers, les cuisiniers ou les tailleurs français) peuvent espérer trouver un emploi. Mais les imprimeurs, les mécaniciens, les charpentiers ou les maçons sont au chômage. Et les exemples d'échecs cuisants abondent. L'ancien député Rouet perd toutes ses économies en montant un music-hall ${ }^{3}$. Un groupe essaye de mettre sur pied une coopérative de consommation, «La Sociale», qui ne dure pas. Gustave Lefrançais, qui avait fondé en France le premier syndicat d'enseignants socialistes, ne reste qu'une année à Londres : impécunieux au point de ne pas pouvoir se payer un logement, il passe la plupart de ses nuits dehors, ou dans les pubs près des docks. ${ }^{4}$

6 Cette misère des réfugiés français, tout comme leurs convictions socialistes fréquentes, en convainquit certains que le gouvernement anglais voulait délibérément les affamer. Pierre Leroux, un socialiste, dont la femme et les enfants vivaient dans un taudis, «sans air», écrivait-il, et "presque sans pain ", dénonçait la prétendue hospitalité de l'Angleterre: "la liberté politique, mais aussi la liberté de mourir de faim $»^{6}$. Parfois, les réfugiés donnaient des leçons de français. Mais il n'était pas facile de trouver ces leçons, ni de les garder. D'abord, parce qu'enseigner le français exigeait quelques rudiments d'anglais qu'eux-mêmes n'avaient pas toujours; Lefrançais expliquait qu'il enseignait le français à l'aide des objets qui composaient le salon de son élève. Ensuite, parce que les Anglais intéressés par ces leçons appartenaient surtout aux classes aisées, peu susceptibles de sympathie pour les idées socialistes de leurs professeurs. Et il y avait la concurrence des domestiques français, plus respectables. Pierre Leroux perdit ses leçons 
quand ses deux élèves découvrirent qu'il était socialiste, tandis que Martin Nadaud dissimula ses origines sociales et politiques auprès de l'école de Wimbledon où il fut embauché, après avoir échoué à travailler comme maçon, sa profession d'origine. ${ }^{7}$ Une ultime difficulté était que, même quand ils cachaient leurs opinions politiques, leur apparence en disait long. Il y avait le stéréotype du républicain, français en particulier, qu'on voit par exemple dans ces dessins de Punch (cf. illustrations n ${ }^{\circ} 1$ et $n^{\circ} 2$ ) : la barbe, la moustache, un longue veste usée, des brochures séditieuses, etc. Louis Blanc écrivait que jusqu'à la guerre de Crimée, la moustache était un attribut du socialiste français. Marx lui-même vécut misérablement, et ne tenait que grâce à l'aide régulière de son ami Engels, dont le père possédait une fabrique à Manchester. Parmi les réfugiés continentaux, ils furent parmi ceux qui y restèrent le plus longtemps, jusqu'à leur décès. Mais ils ne furent jamais intégrés à la bonne société anglaise, à la différence d'un Louis Blanc, par exemple, ou de réfugiés italiens, peut-être ceux qui furent le mieux assimilés.

Illustration $\mathrm{n}^{\circ} 1$. Punch, vol. XIV, 29 April 1848, p. 178

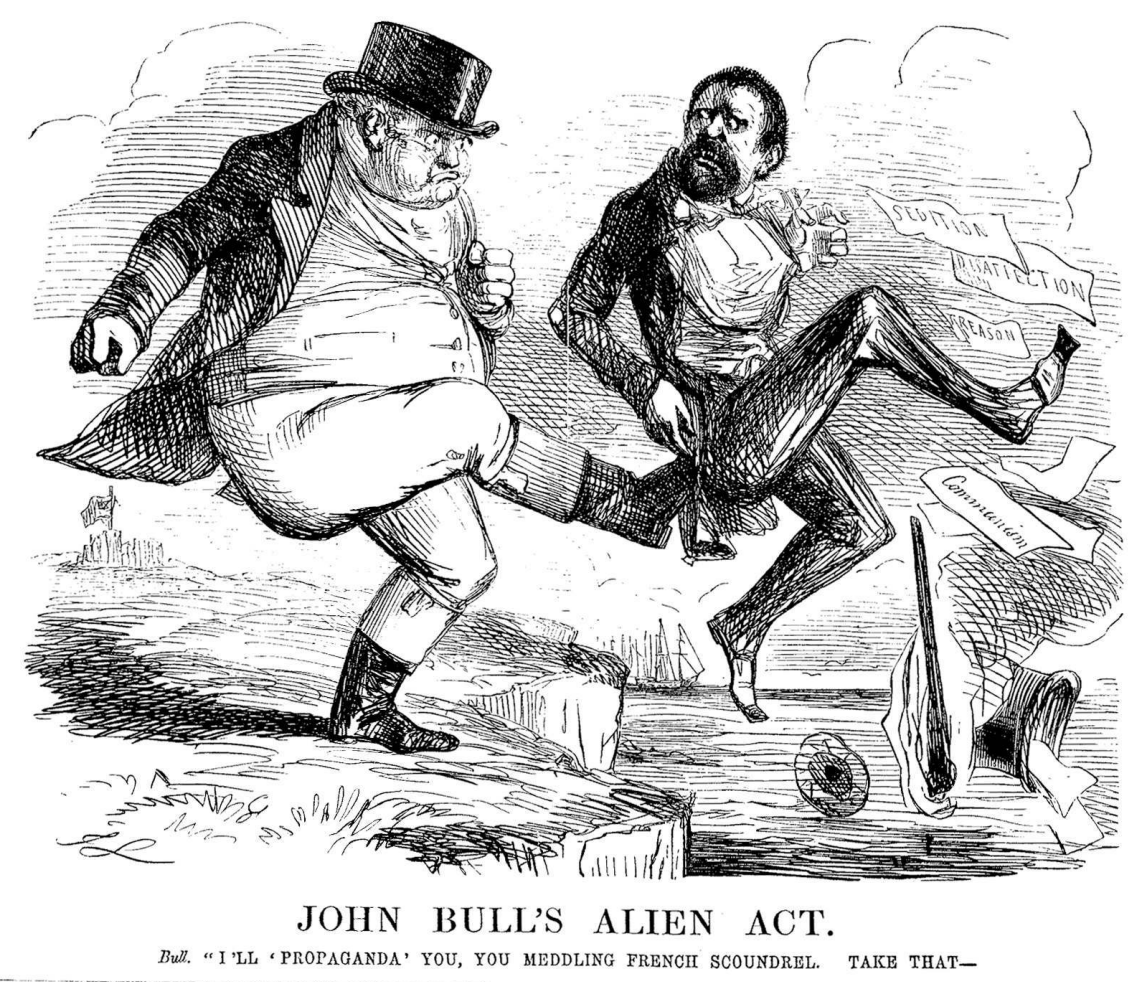




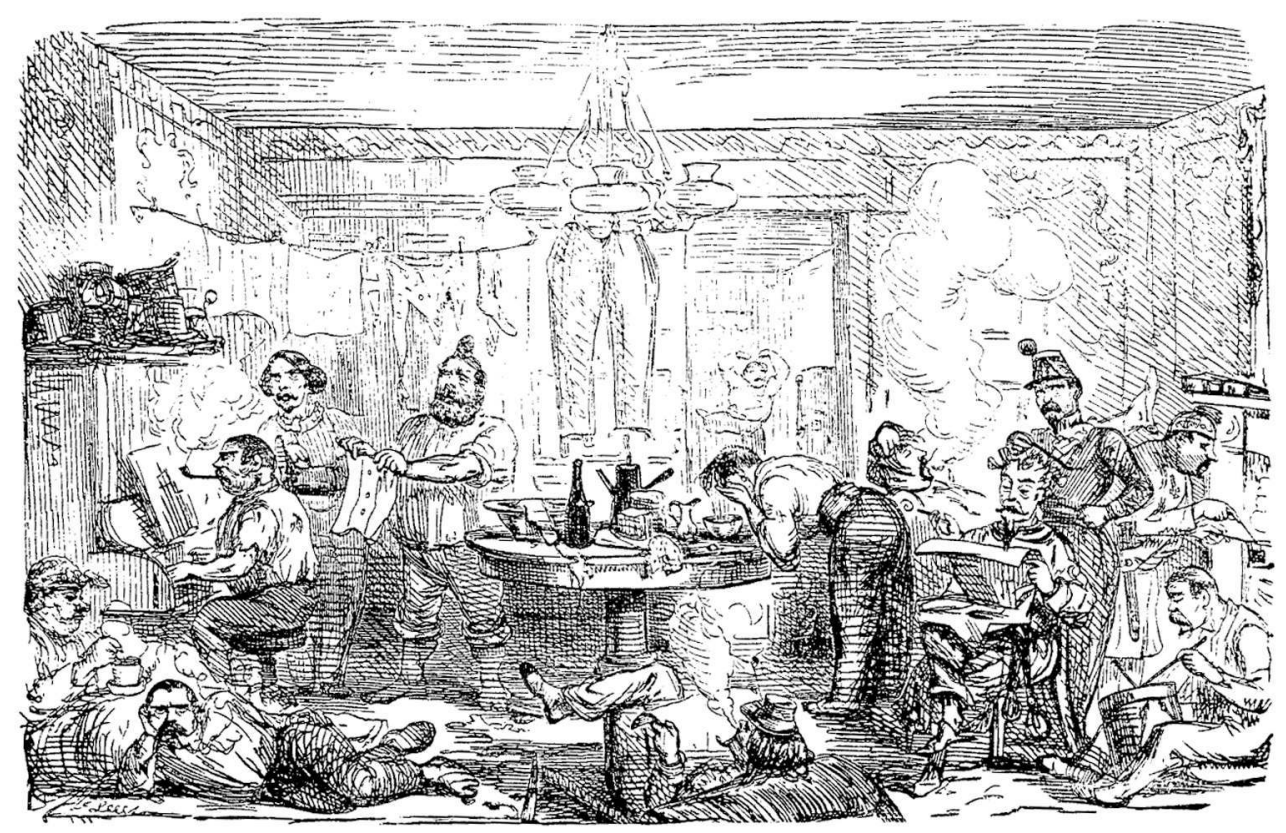

PERFIDIOUS ALBION LETS HIS DRAWING-ROOM FLOOR TO A DISTINGUISHED FOREIGNERTHE RESULT!!

7 Dans l'ensemble, donc, les réfugiés constituent une communauté qui vit difficilement. Ou plutôt des communautés, car la barrière de la langue, le vécu commun, les projets, les discussions interminables sur les situations politiques respectives, rattachent surtout les réfugiés à leurs compatriotes d'exil.

\section{Les réfugiés et le pouvoir britannique}

8 Une deuxième question est celle de l'attitude du pouvoir envers cette communauté. Car les réfugiés n'étaient pas bien vus par tout le monde. D'abord, il y avait l'expression d'une xénophobie traditionnelle - en 1851, par exemple, Punch se plaignait (en blaguant, comme toujours) qu'il y avait maintenant à Londres presque plus d'étrangers que d'Anglais ${ }^{8}$. Mais surtout, il y avait les problèmes politiques posés par les réfugiés. Ainsi, en particulier en période d'agitation, on redoutait dans les milieux conservateurs que les exilés ne viennent contaminer la classe ouvrière anglaise, loyale naturellement, mais à protéger de néfastes influences étrangères. En 1851 même, une des principales craintes formulées à propos de la Great Exhibition était le risque de violence, voire de complot organisé par les républicains. Wellington, par exemple, exprima ces craintes, sans parler d'autres peurs encore plus farfelues, comme celle de l'aéronaute Graham, alors célèbre, qui, sans rire, écrivit au Home Office pour le mettre en garde contre le complot prétendument ourdi par 90000 réfugiés qui mettraient le feu à leurs habitations, et embraseraient la capitale, avec l'aide de 200000 catholiques irlandais déguisés en vendeurs d'allumettes ${ }^{9}$. On ironisa sur cette peur ( $\mathrm{cf}$. illustration $\mathrm{n}^{\circ} 3$ ), et la menace posée par les réfugiés pour la stabilité britannique ne fut plus évoquée. 


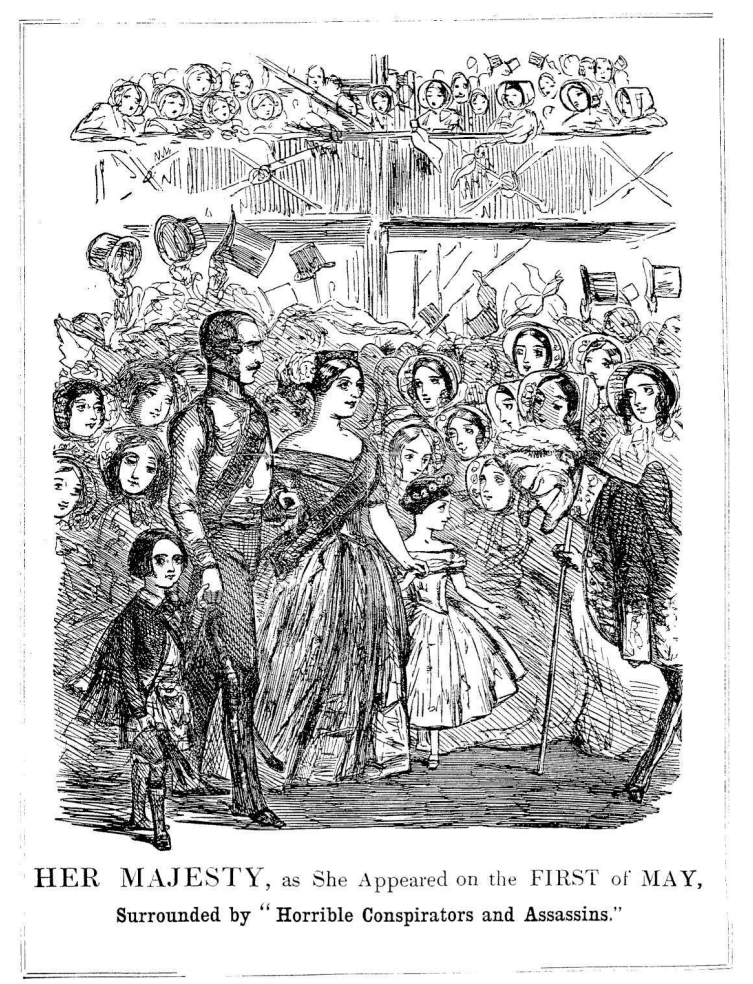

Plus problématique était la pression des régimes des puissances continentales. En effet, Paris, Berlin, Vienne, St Pétersbourg s'accommodaient fort mal du fait que Londres fût devenu le centre de la révolution européenne. Les exilés, les proscrits y intriguaient, complotaient contre ces régimes, publiaient une presse qui traînait dans la boue les hommes forts de l'Europe. Et régulièrement, ils demandaient à Londres d'expulser les exilés, de les livrer. Et on peut s'interroger sur les raisons pour lesquelles le Cabinet n'expulsa ni ne refoula aucun des réfugiés qui se présentaient à ses portes. Ce fut le cas pendant toute la période victorienne. A l'époque, cette attitude constituait une exception remarquable. Pourtant, l'Angleterre n'avait guère d'intérêt matériel à accueillir ces bannis. Et elle aurait eu quelque intérêt diplomatique à en refuser ou à en expulser certains. Pourquoi ne l'a-t-elle pas fait?

Premier élément de réponse : la Grande-Bretagne était alors suffisamment puissante pour dire non; des États plus faibles accueillirent également des réfugiés, mais étaient plus sensibles à la pression des grands États. Deuxième aspect: les relatives libertés d'expression et d'opinion dont jouissait alors le Royaume-Uni par rapport aux régimes autoritaires continentaux. Même des hommes d'État aussi peu démocrates que Palmerston avaient une certaine aversion pour les despotismes européens. Troisième explication, la plus importante: l'opinion publique britannique manifestait alors un intérêt nouveau et considérable pour les affaires internationales. Et elle était farouchement opposée à l'absolutisme qui prévalait, en particulier dans les années 1850, sur le continent. Expulser les réfugiés aurait équivalu à céder aux exigences de personnages aussi impopulaires outre-Manche que Louis-Napoléon Bonaparte. Et, à cela, l'opinion anglaise était résolument opposée. De nombreux éléments en attestent. Quand, en septembre 1850, l'Autrichien le baron Haynau, qui avait écrasé l'insurrection hongroise, fut reconnu à Londres, la foule s'attaqua violemment à lui. A l'inverse, Kossuth 
en 1851 et surtout Garibaldi en 1864 bénéficièrent d'un accueil triomphal, avec des foules considérables se déplaçant pour les accueillir, surtout dans le cas de l'Italien. Autre exemple : l'affaire Orsini. En 1858, en effet, un attentat organisé par l'ancien réfugié Felice Orsini contre Louis-Napoléon Bonaparte fit huit morts et de nombreux blessés. Les bombes avaient été fabriquées en Grande-Bretagne. Napoléon III était furieux, et Londres apparaissait clairement comme responsable de la chose ; on redoutait en Angleterre des représailles françaises, ainsi d'ailleurs que des officiers français en exprimèrent publiquement la menace. Bref, il fallait faire quelque chose. Downing Street chercha à durcir la législation.

11 Le projet de loi proposé provoqua une immense manifestation d'opposition qui fit reculer le gouvernement. Il y eut ensuite un procès, où était notamment jugé le réfugié Simon Bernard, qui avait fourni à Orsini des grenades utilisées lors de l'attentat - Bernard fut donc jugé pour meurtre par un jury populaire, lors d'un procès qui captiva l'intérêt général. Sa culpabilité l'aurait sans doute conduit à la potence. Or le jury populaire le relaxa, au motif que Bernard ne savait pas que les grenades qu'il avait fournies à Orsini... étaient destinées à cet attentat particulier, et que l'Angleterre ne devait pas céder à 600000 baïonnettes françaises. Le gouvernement, qui était partie civile dans cette affaire, renonça à toute poursuite. Les radicaux anglais et les réfugiés eux-mêmes virent cette victoire comme celle du peuple contre l'absolutisme.

Cela n'empêcha pas le pouvoir britannique de s'intéresser de près aux activités des réfugiés. En particulier, un agent, John Sanders, fut chargé de surveiller à plein temps leurs activités. Sanders se faisait passer pour un réfugié lui-même, et participait à leurs réunions, etc. Ses rapports étaient fort précieux; ils étaient transmis au Cabinet, à la Cour, aux ambassades londoniennes des pays amis, aux ambassades anglaises à l'étranger, etc. Bernard Porter les a dépouillés, et a constaté que si Sanders dénonçait quelquefois la véhémence des réfugiés de Jersey, il était extrêmement protecteur à l'égard de ceux de Londres. "Ils sont pauvres, ils respectent la loi, ils sont inoffensifs, etc. " sont les conclusions qui émanent le plus souvent de ces rapports, à tel point qu'il n'est pas exclu que Sanders ait été un agent double, travaillant pour le gouvernement britannique, mais rallié aux réfugiés qu'il surveillait. On reviendra sur cette relation des réfugiés avec la police.

Il n'y eut en tout et pour tout qu'une expulsion au cours de cette période, et encore ce n'en fut pas vraiment une. En 1855, lors de la guerre de Crimée et de l'alliance francoanglaise, le réfugié Félix Pyat, qui résidait à Jersey, stigmatisa la reine Victoria pour son entente avec Napoléon III, dans un discours reproduit dans la presse. « Oui, disait Pyat à l'intention de Victoria, vous avez TOUT sacrifié : la dignité de la reine, la délicatesse de la femme... TOUT - MÊME la CHASTETÉ - pour l'amour de cet Allié». Ces figures de rhétorique étaient acceptées dans le discours politique français, mais moins en Angleterre. Cela déclencha un tollé, et le gouvernement procéda à l'expulsion d'une quarantaine de Français (dont Victor Hugo). Cependant, cette expulsion fut plus symbolique qu'autre chose, car les réfugiés allèrent à Guernesey ou à Londres.

Mais dans l'ensemble, le gouvernement britannique a toléré les réfugiés sur son sol, pour les raisons mentionnées. Et ce sont plus les difficultés matérielles évoquées ou les changements politiques dans le pays d'origine qui conduisaient les réfugiés à rentrer, quand ils n'émigraient pas en Amérique, comme le firent certains. 


\section{La vie sociale et politique des réfugiés}

15 La vie sociale et politique de ces réfugiés est avant tout marquée par l'exil politique et tout ce qu'il comporte de douleur et de difficultés. Les proscrits, les exilés avaient en général joué un rôle local ou national dans les révolutions de 1848. Ils avaient connu l'euphorie des bouleversements politiques, et ils se retrouvaient, pour une période indéterminée, isolés, sans audience, sans rôle.

Beaucoup se convainquaient que le régime qui les avait chassés allait s'effondrer incessamment. Et, pour un Karl Marx qui voyait que la période avait changé, et qu'il fallait se préparer à des années d'une impuissance que compenserait le travail théorique accompli, il y avait d'innombrables militants impatients.

Le premier élément de cette vie sociale et politique reconstituée, c'est la réunion, le club dans une taverne enfumée de Soho ou de Leicester Square, où les réfugiés avaient leurs rues, leurs boutiques, leurs clubs, leurs amis, leurs discussions. Dans ces réunions, on refaisait les journées de juin 1848, on renversait Louis-Napoléon Bonaparte, on s'invectivait, on évoquait les amis communs laissés au pays ou disparus.

Il y avait les nombreuses occasions plus ou moins rituelles: les banquets, les anniversaires, en particulier ceux de la révolution de février 1848, avec des centaines voire un millier de participants, des banderoles républicaines, des bonnets phrygiens, des drapeaux, et tous les symboles républicains. En témoigne l'invitation (cf. illustration ${ }^{\circ}{ }^{4}$ ) à une 'Tea Party' organisée en 1853 en l'honneur de Robespierre.

19 Parmi les invités, on reconnaît des noms français comme ceux de Louis Blanc, Nadaud, Schoelcher, Ledru-Rollin, ceux d'anciens chartistes et de radicaux anglais (G.W.M. Reynolds, Julian Harney, William Linton, Ernest Jones, Bronterre O'Brien), celui de Kossuth, etc. Robespierre était un des héros de ces réfugiés, une référence commune.

20 Le radical anglais Holyoake avait prénommé son fils Maximilien Robespierre en juin 1848 ; quant aux prénoms des fils de G.W.M. Reynolds, c'étaient respectivement Kossuth, Mazzini et Ledru-Rollin. Il y avait également les funérailles, les longues processions avec drapeaux et banderoles, qui se terminaient par d'émouvants discours au cimetière ; ainsi, en avril 1857, quelque 10000 personnes suivirent le cercueil de Rougée.

21 Cela dit, l'exil, surtout à mesure qu'il se prolongeait, c'était aussi l'échec d'un combat, voire d'une vie, et les aigreurs, les rancunes, les haines anciennes ou nouvelles resurgissaient. L'agent Sanders, dépêché dans les réunions de proscrits, observait en 1852 : '[it is a] very curious sight to see the uproar that exists at all times' [...] 'the words Canaille, Voleur, Brigand, Coquin, Jean-Foutre [being] continually used in speaking of each other ${ }^{10}$.

22 En effet, les conflits entre réfugiés étaient fréquents, et tournaient parfois au drame. Ainsi, le réfugié Emmanuel Barthélémy tua un de ses compatriotes réfugiés dans un duel en $1853^{11}$. Il tua ensuite, pour des motifs peu clairs, deux Anglais dans Warren Street, ce qui lui valut la pendaison publique en 1855, et une effigie en cire dans la Chambre des Horreurs de Madame Tussauds - à n'en pas douter, une marque de reconnaissance de la société victorienne ${ }^{12}$. D'autres réfugiés se sont mis au service du régime qui les pourchassait, ce qui était une façon d'être de nouveau écouté.

23 Les réfugiés se regroupaient par affinités politiques. La plupart de ces regroupements étaient nationaux. Il y eut bien quelques regroupements transversaux. 


\section{ROB E SPIERRE. TEA PARTY PUBLIC MEETING THE NATIONAL HALL, HOLBORN, On WFDNESDAY, April 13th, 1853, ROB E S I ER R . \\ Messrs. Inouis Blanc, Madaud, and \\ c. W. In. Reynolds, have protnised in attind. Messrs. Inouis Kossuth, Iedru IRollin, Saffi, Sohoelcher, George Dawson, Gam- mage, G, J. Harney, G. J. Eubyoatis, Dhneat Jones, Iinton, kivingstose, and Rd. Iiloore have been invited.

Source: Bishopsgate Institute, London, G.J. Holyoake Diary, 1853, in Margot Finn, After Chartism. Class and Nation in English Radical Politics, 1848-1874. Cambridge University Press, 1993, p. 123

Ainsi, parmi les non-socialistes, Ledru-Rollin travailla-t-il avec Mazzini et Kossuth. A gauche, parmi les Français, on peut évoquer le groupe de la Commune Révolutionnaire, inspirée par le blanquisme. Entre ces deux tendances, Louis Blanc, Pierre Leroux et Cabet constituèrent une Union Socialiste qui avait ses bureaux à Bloomsbury. Mais, faute de perspectives, ces regroupements ne duraient pas. Les intellectuels comme Louis Blanc ou Ledru-Rollin, préféraient se consacrer à leurs travaux plutôt qu'à d'interminables réunions sans perspectives. D'une certaine manière, des œuvres aussi diverses que $L e$ Capital, Les Misérables et L'Histoire de la Révolution française de Louis Blanc furent le fruit de l'exil outre-Manche. Quant aux plus démunis des réfugiés, ils cherchaient à rentrer au pays; pour les Français, cela fut possible avec l'amnistie de 1859, que certains n'attendirent parfois pas, préférant les geôles françaises à l'asile anglais. Et la création, en 1864, de l'Association Internationale des Travailleurs, ne peut que très partiellement être attribuée aux réfugiés de Londres.

\section{Regards et représentations}

Enfin, on peut évoquer les échanges, les transferts culturels, les représentations, les regards croisés entre réfugiés et Britanniques. La visibilité des réfugiés n'était pas la même à l'époque qu'aujourd'hui. Ainsi, aujourd'hui, Karl Marx est de loin le plus connu des réfugiés - à l'époque, Marx était un inconnu en Angleterre, à la différence de Kossuth ou de Ledru-Rollin. Les échanges directs entre Britanniques et réfugiés ont été assez limités : c'était une communauté petite, numériquement en déclin, assez repliée sur elle- 
même, qui se sentait souvent mal accueillie, dont la plupart des membres ne parlaient pas anglais, du moins à leur arrivée, et qui ne ressentaient pas nécessairement le besoin de connaître des Anglais autrement que pour des raisons matérielles. Néanmoins, interaction et transferts il y a eu. Les réfugiés ont notamment été en rapport avec les nombreux chartistes et radicaux qui leur sont venus en aide. Une boutique montée par le radical Holyoake dans Fleet Street fit office de centre culturel, avec ses salles de réunion, sa librairie, sa bibliothèque ${ }^{13}$. Les formes d'entraide et de relations étaient donc diverses : secours matériel, travaux d'édition, cérémonies du type de celle évoquée pour Robespierre, discussions sur le programme - Gregory Claeys, Margot Finn, ou Miles Taylor ont souligné l'influence des révolutions de 1848 et du socialisme continental sur l'internationalisme de certains chartistes ${ }^{14}$.

Du côté des élites londoniennes, on était nettement plus circonspect. On ironisait beaucoup sur ces réfugiés barbus, hirsutes et un peu effrayants. On partageait souvent l'idée que lors des révolutions de 1848 , la stabilité britannique avait failli être mise en péril à cause de fauteurs de trouble comme eux. 1848 n'avait pas renversé la constitution britannique, mais avait constitué une menace. Et, par exemple, si le coup d'État de décembre 1851 avait été désapprouvé dans les cercles dirigeants britanniques en raison des craintes attachées au bonapartisme, des hommes aussi différents que Palmerston et Bagehot s'étaient réjouis du rétablissement d'un pouvoir fort, supposé être le seul adapté au caractère français. Les proscrits, plus encore que la majorité des Français, étaient considérés comme violents, mais aussi comme versatiles. Avec les années, on se moquerait d'eux, plus qu'on ne les redouterait, ainsi qu'en témoigne un diptyque paru dans Punch en 1858 (cf. illustrations n ${ }^{\circ} 5$ et $6:$ A discussion forum).

Il y eut en fait très peu de réfugiés qui furent acceptés par la bonne société londonienne. Louis Blanc est sans doute le seul exemple français. Dès le début de son séjour à Londres, il fut reçu à dîner dans les élites littéraires : par exemple, par Thomas Carlyle ${ }^{15}$, George Eliot ${ }^{16}$, Herbert Spencer ${ }^{17}$, etc. ${ }^{18}$ Ses conférences sur la France du XVIII ${ }^{\mathrm{e}}$ siècle, en 1860, furent un grand succès mondain, avec des participants comme Dickens ou Thackeray. Enfin, Louis Blanc établit une relation très cordiale avec John Stuart Mill ${ }^{19}$. Mais cette proximité était l'exception plutôt que la règle. Elle tenait largement à la francophilie de John Stuart Mill, qui partageait alors sa vie entre Londres et la France. Et dans l'ensemble, les élites londoniennes ne fréquentaient guère les réfugiés. Ainsi en alla-t-il, par exemple, de Ledru-Rollin, qui ne semble pas avoir attiré (ni recherché) d'amitié britannique. La publication, un an seulement après son arrivée, de l'ouvrage De la décadence de l'Angleterre contribua probablement à son isolement, tout comme son soutien avéré à la cause irlandaise - le genre de choses alors inacceptable dans la bonne société londonienne. 


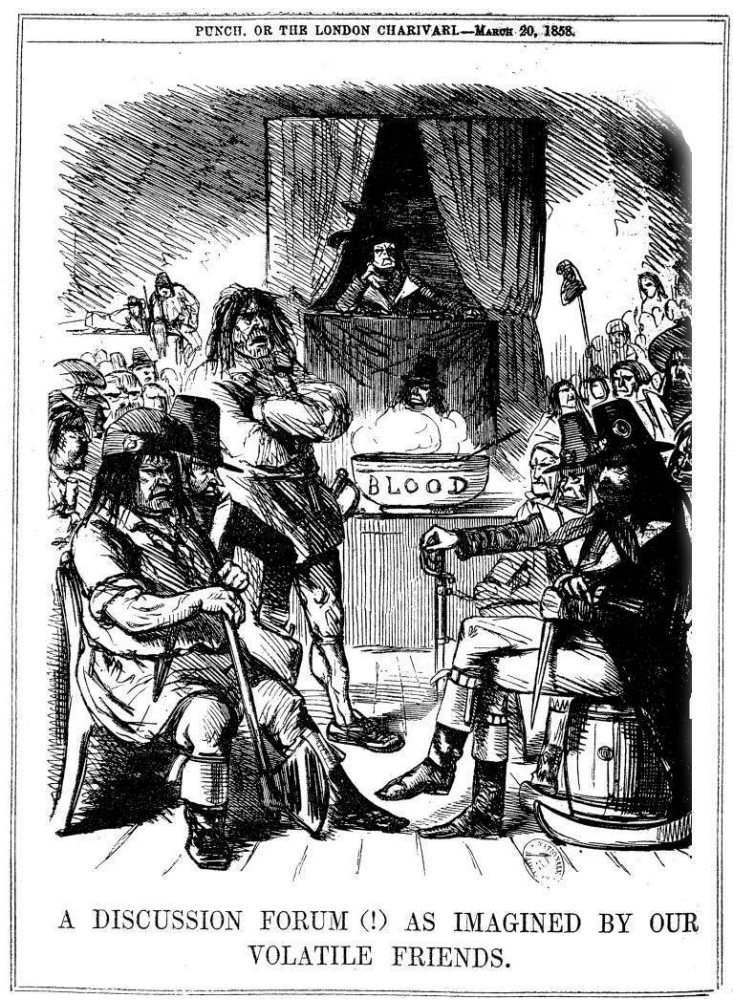

Illustration n6. Punch, vol. XXXIV, 20 March 1858, p. 118

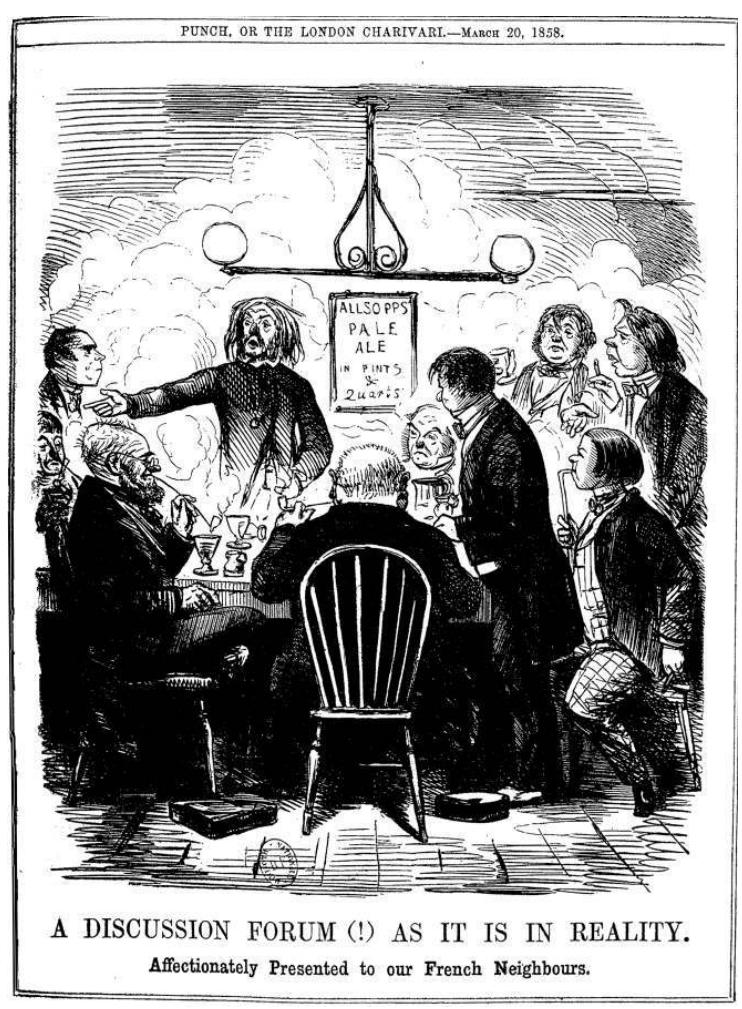

28 Il reste à évoquer le regard global de l'opinion publique, ou des opinions publiques. D'abord, l'immense majorité des Britanniques ou des Londoniens n'avaient pas de contact 
avec les exilés. Peut-être n'y avait-il pas particulièrement de sympathie pour eux ${ }^{20}$. Mais en même temps, l'opinion anglaise ne suivit pas les autorités, la presse, et certains cercles politiques qui cherchaient à exacerber les sentiments xénophobes ou anti-socialistes. Cette propagande échoua en 1848, lors de l'Exposition de 1851, de l'affaire Pyat en 1855 et de l'affaire Orsini en 1858. Aucune véritable manifestation n'eut lieu contre les réfugiés ${ }^{21}$. En revanche, il y eut de grandes réactions populaires pour les défendre, en 1858 en particulier. La motivation première de ces protestations était sans doute la haine des despotes continentaux: en manifestant en faveur des réfugiés, les Britanniques défendaient ce qu'ils considéraient comme un droit fondamental pour les victimes de l'oppression politique: vivre et rester actifs en Angleterre. Donc, les réfugiés furent considérés comme un groupe d'agitateurs par les autorités, comme des braillards insolents par Punch ou le Times, mais surtout comme les victimes de régimes autoritaires par la grande masse des Anglais qui connaissaient leur sort.

A la fin des années 1850, les réfugiés étaient bien moins nombreux. Nombre d'entre eux avaient pu retourner au pays, ou émigrer outre-Atlantique ; les derniers exilés français devaient rentrer en 1870, avec la chute du second Empire, mais ils avaient été précédés par l'essentiel des anciens proscrits. Ceux qui restaient dans les années 1860 étaient souvent plus intégrés, et ne constituaient plus vraiment des communautés comparables à ce qu'ils avaient été dans les mois et les années suivant leur arrivée. Ils ne représentaient plus non plus un enjeu dans la vie politique anglaise. À l'instar de Martin Nadaud, qui était revenu brièvement en France avant de repartir en Angleterre, certains réfugiés s'étaient intégrés à la société anglaise. Pour eux, le retour tant attendu pouvait être un échec, comme ce serait le cas pour Louis Blanc, qui se trouverait à Versailles pendant la Commune de Paris. En revanche, rester en exil pouvait devenir un titre de gloire, posthume notamment, comme ce fut le cas pour Kossuth. Sans doute des travaux plus poussés permettraient-ils de mieux suivre les destinées de réfugiés obscurs. Un autre angle d'approche serait celui des représentations de la ville victorienne que construisaient les réfugiés. Cela nous ramène au thème de ce numéro. Les réfugiés étaient aux marges de Londres, à l'intersection des marges nationales et des marges politiques. Avec le plus souvent, comme principale perspective, non pas d'intégrer la société anglaise, mais de rentrer, de retrouver le rôle que l'exil leur refusait.

\section{NOTES}

1. Bernard PORTER, The Refugee Question in mid-Victorian Politics, Cambridge University Press, 1979.

2. En 1999, le German Historical Institute de Londres a organisé un colloque qui a permis d'aborder plus en détail ces parcours Cf. Sabine FREITAG (ed.), Exiles from European Revolution: Refugees in Mid-Victorian England, Oxford \& New York: Berghahn Books, 2003.

3. Alexandre ZÉVAES, «Les Proscrits français en 1848 et 1851 à Londres », La Révolution de 1848, tome XX, Paris, 1923, p. 351.

4. Gustave LEFRANÇAIS, Souvenirs d'un révolutionnaire, Paris : Futur antérieur, 1972. 
5. "Je pensais à ma femme et à mes enfants enfermés depuis deux mois, sans air et presque sans pain, dans un taudis à Londres ». Pierre LEROUX, La Grève de Samarez, poème philosophique, Paris : Dentu, 1863, vol. I, p. 237.

6. "Ô hospitalité de l'Angleterre : tu te montras là ce que tu es : la liberté politique, mais aussi la liberté de mourir de faim. Quel secours Albion donna-t-elle à tant de victimes? Aucun, absolument aucun.»Ibid., p. 305.

7. Martin NADAUD, Mémoires de Léonard ancien garçon maçon, Paris : Hachette, 1976, p. 439. Sur Martin Nadaud, on lira également avec intérêt Gillian TINDALL, Le Voyage de Martin Nadaud, Paris : Éditions du Rocher, 2001 (1999).

8. 'People have cried out that there were no foreigners in London; yet London never was so full of them as at the present moment. We almost believe there are more foreigners in our metropolis just now than Englishmen'. Punch, 20 September 1851, vol. XXI, p. 135.

9. Cf. Bernard PORTER, op. cit., p. 86.

10. Police Report (Sanders), 14 October 1852, PRO, HO 45/4547A. Cité in Bernard PORTER, op. cit., p. 27.

11. The Times, 22 March 1853, p. 7.

12. The Times, 9 December 1854 , p. 10 ; 5 January 1855, p. 9 ; 11 January, p. 9 ; 17 January, p. 10 ; 23 January, p. 8.

13. Margot FINN, After Chartism : Class and Nation in English radical Politics, 1848-1884, Cambridge University Press, p. 117.

14. Gregory CLAEYS, Citizens and Saints : Politics and Anti-politics in early British Socialism, Cambridge University Press, 1989, pp. 268-274 ; Margot FINN, op. cit. ; Miles TAYLOR, The Decline of British Radicalism, 1847-1860, Oxford University Press, 1995.

15. Voir la description très élogieuse de Louis Blanc par Carlyle, par exemple in James FROUDE, Thomas Carlyle : A History of his Life in London, 1834-1881, London: Longmans \& Green, 1884, vol. I, p. 452.

16. Par exemple, cf. lettre de George Eliot à M. et Mme Charles Bray, 27 avril 1852 : 'We had a brilliant soirée yesterday evening. W. R. Greg Foster (of Rawden), Francis Newman, the Ellises and Louis Blanc were the stars of the greatest magnitude.' George ELIOT, The Letters of George Eliot, Oxford University Press, 1954, vol. II, p. 21.

17. Cf. Herbert SPENCER, Autobiographie, Paris : Presses Universitaires de France, 1987, p. 25.

18. Par exemple, Charles Greville, le haut fonctionnaire whig passé à la postérité pour son journal privé, y notait le 20 octobre 1848, après un dîner où il avait rencontré Louis Blanc : 'He is very gay, animated, and full of information, takes in very good part anything that is said to him, and any criticisms on his revolution and the Provisional Government.' Charles C.F. GREVILLE, The Greville Memoirs : A Journal of the Reign of Queen Victoria from 1837 to 1852, London: Longmans, 1885, vol. III, p. 235.

19. On dispose de nombreux témoignages de l'estime portée par John Stuart Mill à Louis Blanc. Par exemple, en septembre 1848 : 'I feel an entireness of sympathy with them [the Provisional Government] which I never expected to have with any government.... I also sympathise very strongly with such socialists as Louis, who seems to be sincere, enthusiastic, straightforward, and with a great foundation of good sense and good feeling, though precipitate and raw in his practical views.' (lettre à John Pringle Nichol, 30 septembre 1848, in John Stuart MILL, The Earlier Letters of John Stuart Mill 1812-1848, Toronto: University of Toronto Press, 1963, pp. 739-740). Voir aussi : Autobiography, London: Longmans, Green, Reader \& Dyer, 1873, p. 231; et 'Vindication of the French Revolution of February 1848', in John Stuart MILL, Essays on French History and Historians, Toronto: University of Toronto Press, 1985.

20. Bernard PORTER, 'The Asylum of Nations: Britain and the Refugees of 1848' pp. 43-56 in Sabine FREITAG (ed.), op. cit. 
21. La réunion des habitants de Jersey après le discours et l'article de Pyat constitue une exception ; mais ce meeting, à l'initiative duquel se trouvait le Lieutenant-Gouverneur de l'île, n'avait rien de spontané.

\section{RÉSUMÉS}

Dans la foulée de l'échec du «Printemps des peuples », républicains et socialistes affluent à Londres pour échapper à la répression. Ces communautés de réfugiés cherchent à survivre matériellement, mais aussi à reconstituer des groupes politiques, une vie sociale, des liens avec le pays qu'ils ont quitté. Exilés et Britanniques construisent les uns sur les autres de nombreuses représentations.

In the wake of the failure of the 'People's spring', Republicans and Socialists rushed to London to escape repression. These refugee communities strove to survive materially, but also to rebuild political groups, a social life, and links with the country they had left. The refugees and the British built up various images of each other.

\section{AUTEUR}

\section{FABRICE BENSIMON}

Université Paris 10 - Nanterre 